Virtual Mentor. March 2004, Volume 6, Number 3.

doi: 10.1001/virtualmentor.2004.6.3.jdsc2-0403

Journal Discussion

\title{
A Place of Healing? Medical Injuries in the Hospital Setting
}

\section{Medical errors often result in longer hospital stays and increased costs in patient care.}

\author{
Richard Morse, MA
}

Zhan C, Miller MR. Excess length of stay, charges, and mortality attributable to medical injuries during hospitalization. JAMA. 2003;290:1868-1874.

Although the literature pertaining to injuries in medicine has grown steadily in the last 5 years, there are still few studies that are methodologically rigorous. The limited research on medical injuries to date has primarily relied upon medical record abstraction and has been conducted on a limited scale. As Drs Zhan and Miller point out in their recent JAMA article, "Excess Length of Stay, Charges, and Mortality Attributable to Medical Injuries During Hospitalization," part of the reason for the lack of good empirical information about medical injuries is that medical injuries occur during nearly every stage of the health care process. It is often difficult to pinpoint the cause of such injuries definitively. In addition, the absence of a commonly accepted nomenclature in the injury research field helps explain why relatively little is known about the prevalence and financial effects of medical injuries in the United States [1].

In an effort to demonstrate the adverse personal and societal effects of medical injuries, Zhan and Miller assessed the degree to which injuries during hospitalization contribute to excess length of stay, charges, and death. The primary data source for their analysis is a nationwide inpatient sample of approximately 7.45 million hospital discharge abstracts from 994 acute care hospitals [2]. The authors used this data and a set of "Patient Safety Indicators" developed by the Agency for Healthcare Research and Quality to screen for incidents of medical injury [3]. Through extensive empirical analysis, the authors came up with 18 distinct categories of medical injury that they concluded were reasonably specific for the purposes of their analysis.

As the authors thoughtfully conclude, the adequate control of confounding variables is often the major challenge in attributing adverse outcomes correctly to medical injuries. In this study, variables such as principal reason for admission, severity of illness, comorbid conditions, and other patient characteristics understandably affected the length of hospital stay, charges, and mortality. To control for such confounding variables, the authors used a multivariable matching technique by which they matched each medical injury subject with up to 4 controls from the same hospital. Controls shared diagnosis (according to DRG on the chart) sex, age (within 10 years), and membership in a white or nonwhite "racial" group. Through this extensive matching algorithm, the authors attempted to reduce the impact of other clinical factors on the variables being examined.

Based upon their analysis, Zhan and Miller identified a substantial number of categories of injury resulting directly from failures in hospital care, which were likely to result in a longer length of stay, higher charges, and a greater likelihood of mortality. For example, the most serious adverse event appeared to be postoperative sepsis. According to the authors, patients with postoperative sepsis experienced a length of hospital stay that was 10.89 days longer, \$57 727 more expensive, and had a 21.92 percent higher attributable mortality than those of similar controls. In severity of adverse events, postoperative sepsis was followed by postoperative wound dehiscence. Patients with postoperative 
wound dehiscence experienced an excess hospital stay of 9.42 days, were charged $\$ 40323$ more, and had a 9.63 percent higher mortality when compared with controls. Infections acquired during hospitalization were associated with 9.58 extra days in the hospital, \$38 656 in excess charges, and 4.31 percent greater likelihood of mortality.

Because the authors drew their conclusions based upon matched controls, it is difficult to make an accurate national extrapolation of their findings. However, given that their data encompasses discharge records from an approximately 20 percent of US hospitals, one can reasonably infer that the national estimates of the total cost could be up to 5 times higher than the total cost these studies would suggest. If this data is valid nationally, the extrapolation would indicate that these 18 categories of medical injuries could conceivably result in 2.4 million extra days of hospitalization, $\$ 9.3$ billion in excess charges, and 32591 deaths in the US annually [1]. At this level, the excess costs associated with just these 18 injuries would approximate 2 percent of the national health expenditure on total hospital care in this country [5].

While the study contributes little to the already scant knowledge concerning the national prevalence of various types of medical injury, it does provide rather significant information as to the adverse effects of selected medical injuries on patients and on health care resources. For the first time, a study has provided specific estimates on how 18 types of medical injury affect length of hospital stay, cost, and likelihood of mortality. The results of this study clearly demonstrate that injuries in hospitals pose a significant threat to the patient safety and incur substantial costs to society. While the analytical approach of this study may have ultimately limited its national significance, it nonetheless reinforces the need for continued research in the area of medical injury. Most significantly, it is imperative that studies are conducted that assess the prevalence of injuries and develop and test prevention strategies to lessen the effects of these injuries.

\section{Questions for Discussion}

1. After Zhan and Miller's article appeared, many news stories were written about injuries from medical mishaps. Was the media furor justified by the authors' findings, or did the public simply not understand the risks of surgery and other medical procedures?

2. The US Agency for Healthcare Research and Quality posted a list of 20 tips for patients after Zhan and Miller's article was published. One piece of advice was to choose a hospital with a high volume of the type of surgery the patient needed. How practical is such advice in today's managed health care system?

3. According to Zhan and Miller's findings that hospital failures result in longer hospital stays and higher charges, who should pay for this extra care? Should the hospital be held accountable, and if so, how?

\section{References}

1. Zhan C, Miller MR. Excess length of stay, charges, and mortality attributable to medical injuries during hospitalization. JAMA. 2003;290:1868.

View Article PubMed Google Scholar

2. The Agency for Healthcare Research and Quality, Healthcare Cost and Utilization in Project (HCUP). Nationwide Inpatient Sample (NIS). Rockville, MD: Agency for Healthcare Research and Quality; July 2003. Accessed February 23, 2004.

Google Scholar

3. Agency for Healthcare Research and Quality. AHRQ Quality Indicators-Patient Safety Indicators. Rockville, MD: Agency for Healthcare Research and Quality; 2003.

Google Scholar

4. Kohn LT, Corrigan JM, Donaldson MS, eds; Committee on Quality of Health Care in America, Institute of Medicine. To Err is Human: Building a Safer Health System. Washington DC: National Academies Press; 2000. Google Scholar

5. Centers for Medicare \& Medicaid Services Web site. Highlights—national health expenditures, 2002. Accessed February 23, 2004. 
The viewpoints expressed on this site are those of the authors and do not necessarily reflect the views and policies of the AMA.

(C) 2004 American Medical Association. All Rights Reserved. 